\title{
Primer registro de Panochthus Burmeister (Xenarthra; Cingulata; Glyptodontidae) en Perú
}

\author{
First record of Panochthus Burmeister (Xenarthra; Cingulata; Glyptodontidae) \\ in Peru
}

D.O.I.: https://doi.org/10.30550/j.agl/2018.30.1/2

\section{Martín ZAMORANO ${ }^{1}$ y Gustavo JARA ALMONTE ${ }^{2}$}

1 División Paleontología de Vertebrados, Museo de La Plata, Facultad de Ciencias Naturales y Museo, Universidad Nacional de La Plata. Paseo del Bosque s/n,1900 La Plata. Argentina. CONICET. marzamorano@fcnym.unlp.edu.ar

a Colaborador de la colección del Departamento Paleontología de Vertebrados, Museo de Historia Natural de Lima, 14, Av. Arenales 1256, Jesús María, Lima 14, Perú. tavoterror@yahoo.es

Resumen - Panochthus Burmeister es uno de los géneros de gliptodóntidos más ampliamente distribuidos geográficamente en el Pleistoceno del sur de América del Sur. Revisiones sistemáticas recientes revelaron la existencia de siete especies: P. intermedius Lydekker, $P$. subintermedius Castellanos, P. tuberculatus [Owen], P. frenzelianus Ameghino, P. greslebini Castellanos, P. jaguaribensis Moreira y P. hipsilis Zurita, Zamorano, Scillato-Yané, Fidel, Iriondo y Gillette. En esta contribución se describe un osteodermo que proviene del Pleistoceno de la ribera del río Desaguadero, Perú, aproximadamente a 3800 metros sobre el nivel del mar. La presencia, en su superficie externa, de figuras reducidas que siguen un patrón reticular permite atribuirlo a Panochthus. Este es el primer registro de este género en Perúy, de esta manera, se amplía su distribución geográfica conocida.

Palabras clave: Glyptodontidae, Panochthus, Pleistoceno, paleogeografía, Perú.

> Abstract - Panochthus Burmeister is one of the most geographically widespread glyptodontid genera in the Pleistocene of southern South America. Recent systematic revisions revealed the presence of seven species: $P$. intermedius Lydekker, $P$. subintermedius Castellanos, $P$. tuberculatus (Owen), P. frenzelianus Ameghino, P. greslebini Castellanos, P. jaguaribensis Moreira and P. hipsilis Zurita, Zamorano, Scillato-Yané, Fidel, Iriondo and Gillette. An osteoderm from Pleistocene deposits cropping out on the banks of the Desaguadero River, Peru, at about 3800 meters above sea level, is described herein. The presence of a clear reticular pattern on the outer surface of the osteoderm is indicative of Panochthus. This is the first record of Panochthus from Peru and, thus, the geographic range of the genus is extended.

Keywords: Glyptodontidae, Panochthus, Pleistocene, paleogeografía, Peru.

\footnotetext{
- Ref. bibliográfica: Zamorano, M.; Jara Almonte, G. (2018). Primer registro de Panochthus Burmeister (Xenarthra; Cingulata; Glyptodontidae) en Perú. Acta geológica lilloana 30 (1): 23-30.

> Recibido: 30/11/17 - Aceptado: 08/08/18

> URL de la revista: http://actageologica.lillo.org.ar

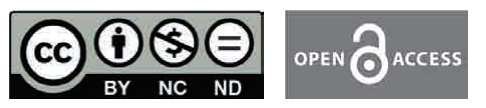

> Algunos derechos reservados. Esta obra está bajo una Licencia Creative Commons Atribución - No Comercial - Sin Obra Derivada 4.0 Internacional.
} 
Los Glyptodontidae (Eoceno tardío-Holoceno temprano) son xenarthros acorazados característicos de América del Sur. En el Plioceno, durante el Gran Intercambio Biótico Americano (GIBA), el grupo alcanzó América Central y América del Norte (Gillette y Ray 1981; Carranza-Castañeda y Miller 2004; Carlini et al. 2008). Los gliptodóntidos constituyen uno de los clados de Cingulata de mayor éxito adaptativo (Zurita et al. 2016). Durante el Neógeno y el cuaternario presentan una importante diversidad y grandes tamaños, (Scillato-Yané 1977; Scillato-Yané y Carlini 1998; Vizcaíno et al. 2011; Soibelzon et al. 2012; Zamorano et al. 2013), junto a una ampliación en su distribución latitudinal. La mayor diversidad está centrada en ámbitos australes, donde el grupo exhibe una importante diferenciación taxonómica con respecto a las formas del norte de América del Sur (Gillette y Ray 1981; Zurita et al. 2016). La comprensión de la evolución biológica y biogeográfica de este grupo se ve limitada por el escaso conocimiento de las formas cuaternarias en latitudes medias en Sudamérica (p.ej., Tejada-Lara et al. 2015). Panochthus Burmeister y Glyptodon Owen son los gliptodóntidos de mayor distribución latitudinal en América del Sur (Zurita et al. 2009, 2016; Zamorano 2012; Zamorano et al. 2014a,b) y representan, a su vez, los dos únicos géneros registrados en las regiones Andina y sub-andina
(Hoffsfetter 1973; Scillato-Yané et al. 1995; Pujos y Salas 2004; Zurita et al. 2011, 2017; Zamorano et al. 2014a).

Panochthus es uno de los géneros de gliptodóntidos más abundantes, grandes, diversificados y ampliamente distribuidos durante el Pleistoceno en América del Sur (véase Fariña 2001; Zamorano 2012; Zamorano et al. 2014a,b, 2015). La última revisión integral de este género (Zamorano et al., 2014a) demostró que incluye seis especies: $P$. intermedius Lydekker, del Ensenadense (Pleistoceno temprano tardío) de la ciudad de Buenos Aires, Argentina y Cochabamba, Bolivia; P. subintermedius Castellanos, del Ensenadense de la ciudad de Buenos Aires; P. tuberculatus (Owen), del Bonaerense-Lujanense (Pleistoceno medio-Pleistoceno tardío) de gran parte de Argentina, Uruguay, sur y centro de Bolivia y sur de Brasil; P. frenzelianus Ameghino, del Bonaerense (Pleistoceno medio-Pleistoceno tardío) de la provincia de Buenos Aires, Argentina y los alrededores de Montevideo, Uruguay; P. greslebini Castellanos, del Lujanense (Pleistoceno tardío) de la provincia de Buenos Aires, Argentina y Pleistoceno del noreste de Brasil; y P. jaguaribensis Moreira, del Pleistoceno del noreste de Brasil; a las que se agregó recientemente P. hipsilis Zurita, Zamorano, Scillato-Yané, Fidel, Iriondo y Gillette, del Pleistoceno de la Cordillera Oriental de Bolivia (Zurita et

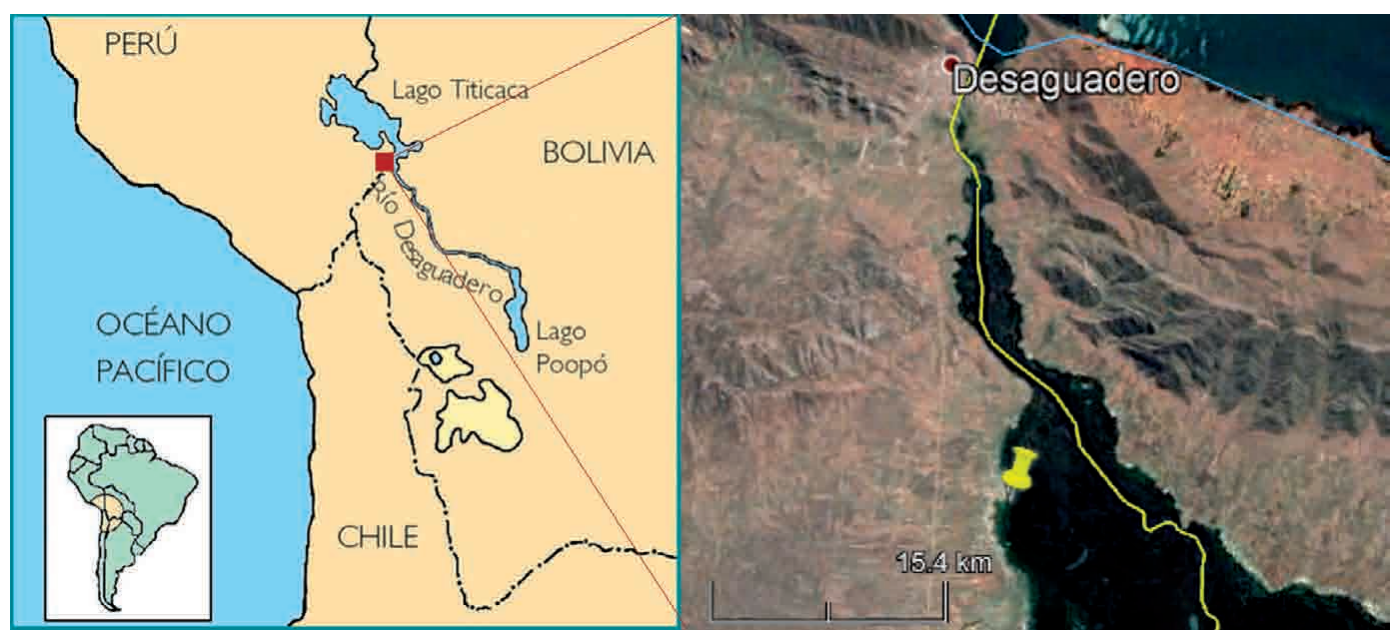

Figura 1. Ubicación geográfica del lugar de hallazgo. 


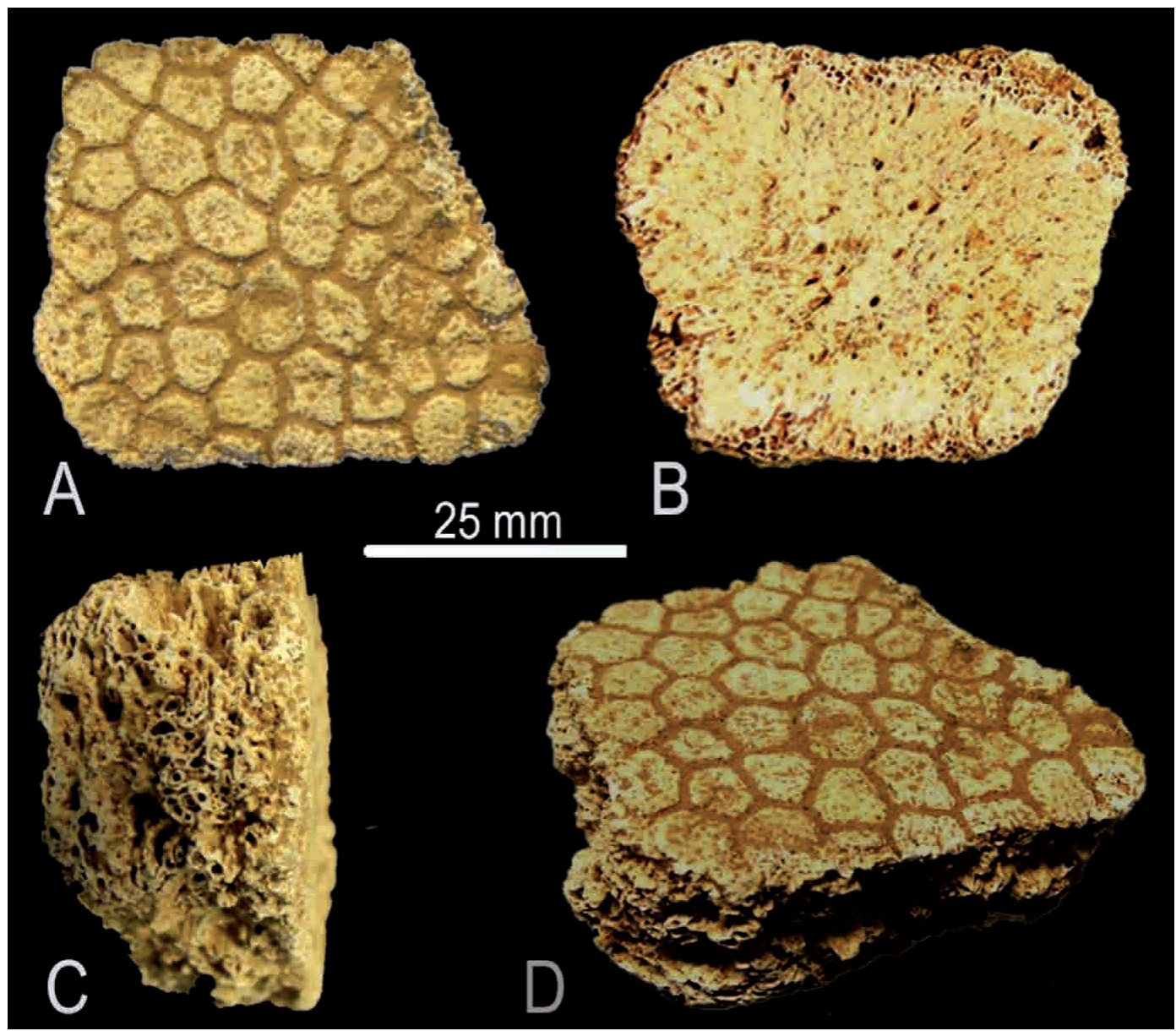

Figura 2. Osteodermo de Panochthus sp. (MUSM 3632). A, vista dorsal; B, vista ventral; C, vista lateral; D, vista general.

al., 2017). Además, Zamorano (2012), Zamorano y Brandoni (2013) y Zurita et al. (2017) señalaron que Panochthus constituye un género monofilético.

En esta contribución se describe por primera vez material de Panochthus de Perú, y de esta forma se amplía el rango de distribución geográfica del género durante el Pleistoceno.

El material estudiado consiste en un osteodermo completo coleccionado en el Pleistoceno de la ribera del río Desaguadero, departamento de Puno, provincia de Chucuito, Perú (S 16³8'2", O 69¹'44"; Figura 1), aproximadamente a $3800 \mathrm{msnm}$. El ejemplar fue hallado el 15 de junio de 2013 por uno de los autores (GJA) y está depositado en el
Museo de Historia Natural de la Universidad Mayor de San Marcos, Lima, Perú, bajo la sigla MUSM 3632.

\section{PALEONTOLOGÍA SISTEMÁTICA}

Familia Glyptodontidae Gray, 1869

Género Panochthus Burmeister, 1866

Especie tipo: Glyptodon tuberculatus

Owen, 1845.

Panochthus sp.

Figuras 2A-D, 3F

Descripción.-El osteodermo presenta un contorno cuadrilátero trapezoide, sus lados miden 44, 37, 34 y 29 mm y el espesor es de $26 \mathrm{~mm}$. Su espesor es menor que el ob- 


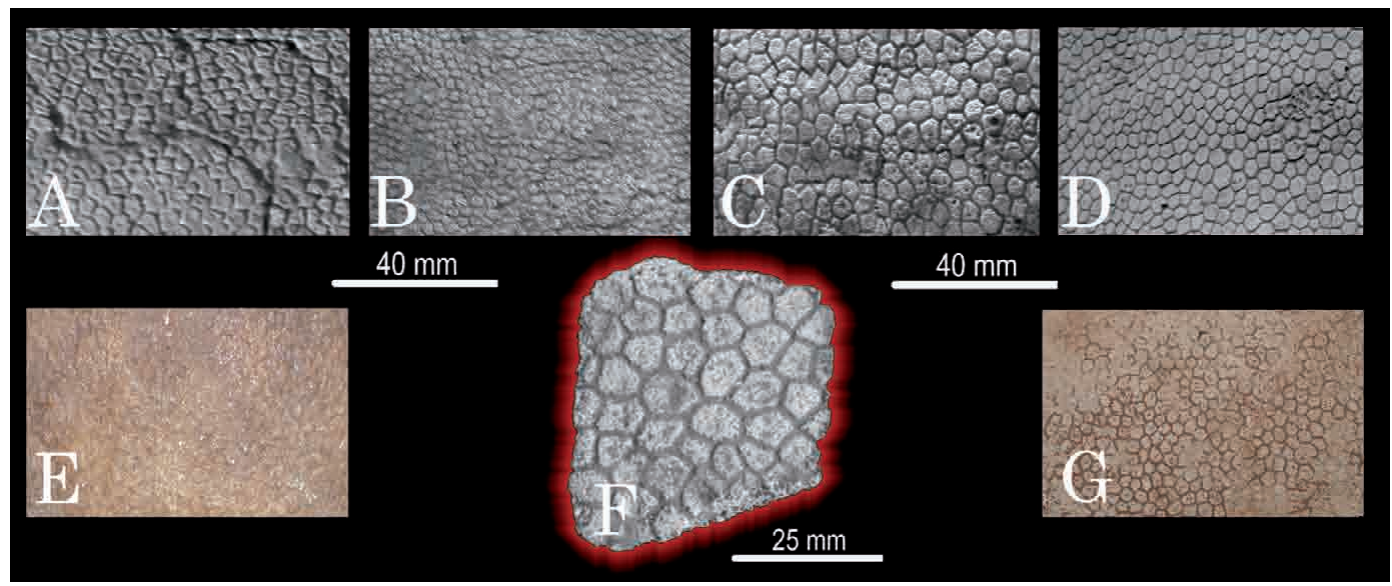

Figura 3. Fragmentos de coraza dorsal. A, Panochthus tuberculatus (MLP 16-29); B, P. frenzelianus (AMNH 11243); C, P. intermedius (MLP 16-36); D, P. subintermedius (MACN-PV 5130); E, P. greslebini (MNRJ 2760); F, Panochthus sp. (MUSM 3632); G, P. hipsilis (MURB 1906B).

servado en las especies de Glyptodon, y mayor que el de Neosclerocalyptus Paula Couto (véase Zurita 2007). La superficie dorsal del osteodermo presenta tubérculos poligonales pequeños y similares en tamaño, distribuidos por toda la superficie, sin un patrón de ordenamiento aparente. Esta misma condición se observa en Panochthus tuberculatus, P. frenzelianus, $P$. subintermedius, $P$. hipsilis, la porción de coraza dorsal conocida de $P$. greslebini, y la región dorsal de la coraza dorsal de $P$. intermedius (Zamorano 2012; Zamorano et al. 2014a; Zurita et al. 2017) (de P. jaguaribensis solo se conocen el tubo caudal y dos fragmentos del escudo cefálico, Porpino et al. 2014), por lo que el material estudiado aquí es asignado a dicho género.

Observaciones.- La ornamentación del osteodermo estudiado muestra cierta similitud con el patrón reticular documentado en la región dorsal de la coraza de algunos ejemplares de Glyptodon reticulatus Owen (Zurita et al. 2018); no obstante, en esta última especie las figuritas son notablemente más grandes y además el espesor de los osteodermos es mayor. La ornamentación de Panochthus sp. también es comparable con la documentada en Neuryurus trabeculatus Zurita y Ferrero. Los osteodermos de la región dorsal de la coraza de $N$. trabeculatus poseen pequeños tubérculos distribuidos uniformemente en toda la superficie, entre los cuales existen perforaciones y forámenes (Zurita y Ferrero 2009). El ejemplar de Perú se diferencia, sin embargo, por carecer de estas perforaciones, y por su menor espesor.

Comparado Panochthus sp. con los restos conocidos de Propanochthus bullifer (Burmeister), las diferencias también son evidentes, ya que en la región póstero-dorsal de la coraza dorsal de esta última especie existe, en cada osteodermo, una gran figura central rodeada de tres a siete hileras de pequeñas figuras periféricas (Zamorano 2013). Asimismo, Burmeister (1874) señaló que los osteodermos de Pr. bullifer son más delgados que los de Panochthus. Por su parte, los géneros Glyptodon, Hoplophorus Lund, Neosclerocalyptus, Nopachtus Ameghino, y Plohophorus Ameghino presentan, en cada osteodermo, una figura central subcircular rodeada de varias hileras de figuritas periféricas subcirculares (Ameghino 1889; Zurita 2007; Porpino et al. 2010; Zamorano et al. 2011; Zurita et al. 2018), diferenciándose claramente del material aquí estudiado.

Los únicos registros de gliptodóntidos cuaternarios en territorio peruano corresponden exclusivamente al género Glyptodon 


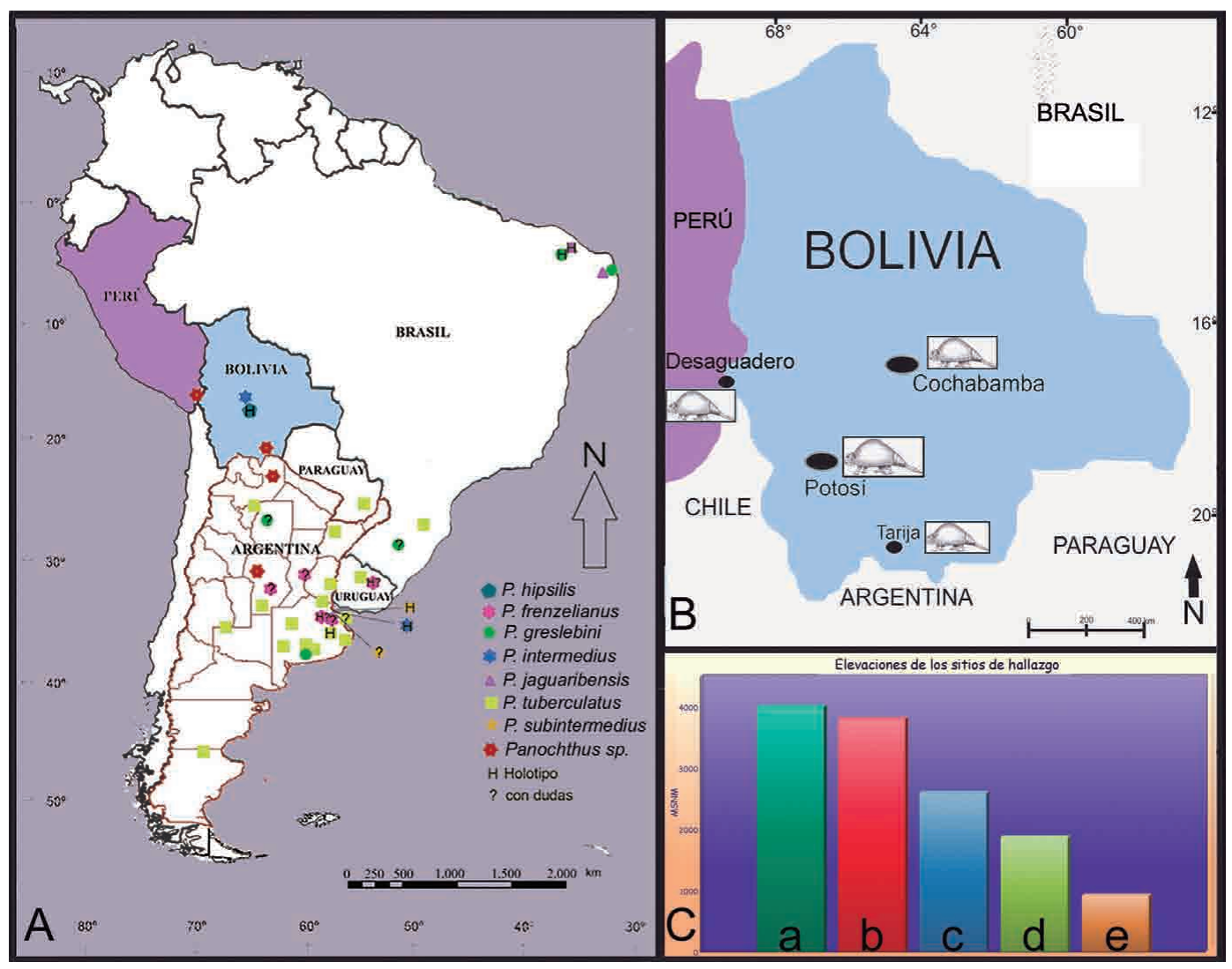

Figura 4. Distribución geográfica. A, de las especies de Panochthus; B, de los registros de Panochthus a mayores alturas sobre el nivel del mar; C, a, alrededores de Potosí (holotipo de P. hipsilis] a 44000 msnm; b, Desaguadero, Perú (atribuido a Panochthus sp.) a 43800 msnm; c, cercanías de Cochabamba, Bolivia (atribuido a P. intermedius] a 42600 msnm; c, Valle de Tarija, Bolivia (atribuido a Panochthus sp.) a 41870 msnm; e, Valle de Traslasierra, Nono, Argentina (atribuido a Panochthus sp.) a 4920 msnm.

(Kalafatovich 1955; Willard 1966; Hoffstetter 1973, 1986; Pujos y Salas 2004; Zurita et al. 2009, 2012; Oliveira et al. 2010); asimismo, Pujos y Salas (2004) comunican que $G$. clavipes Owen solo está presente en el área Cusco-Puno. A su vez, Hoffstetter (1973) señala para la región de Azángora (Puno), material perteneciente a un género no definido, y finalmente para Ymata (Puno), registra un espécimen cf. Panochthus sin brindar datos de estos últimos materiales (véase también Pujos y Salas, 2004).

Tejada-Lara et al. (2015) mencionan materiales de gliptodóntidos (Parapropalaehoplophorus septentrionalis Croft, Flynn y Wyss; Glyptodontidae indet.; Boreostemma sp.; Neoglyptatelus originalis Carlini, Vicaíno y Scillato-Yané [Glyptatelina]) que provienen del Mioceno medio temprano de Fitzcarrald (Perú) y muestran una interesante vinculación de esta fauna con los taxones del Mioceno medio de la Venta (Colombia) compartiendo los géneros Boreostemma Carlini, Zurita, Scillato-Yané, Sánchez y Aguilera, y Neoglyptatelus Carlini, Vicaíno y Scillato-Yané. Esta información resulta esperable dada la configuración paleobiogeográfica y paleoambiental de América del Sur en ese lapso (véase Zurita et al. 2016).

Esta contribución permite confirmar la presencia del género Panochthus en Perú y, de esta manera, se amplía la distribución 
geográfica conocida para este taxón. $\mathrm{Pa}$ nochthus se distribuyó en las regiones chaco-pampeana, mesopotámica, subandina $\mathrm{y}$ patagónica septentrional de Argentina; Uruguay; sureste y noreste de Brasil; sureste de Paraguay; centro y sur de Bolivia; y sureste de Perú (Figura 4A). Los hallazgos a mayor altura sobre el nivel del mar se verificaron en estas últimas regiones (Figura 4B-C). Su límite de distribución más austral es la localidad de Fitz Roy (S 4701' - O 67²1'), Departamento Deseado, provincia de Santa Cruz de Argentina -P. tuberculatus- (Tauber y Palacios, 2007), y los registros del noreste de Brasil, en el Estado de Ceará

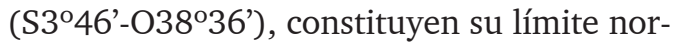
te -P. greslebini y $P$. jaguaribensis - (véase Castellanos, 1942; Moreira, 1965). Mientras que este registro de Perú es su límite más occidental.

\section{AGRADECIMIENTOS}

A Gustavo J. Scillato-Yané y Alfredo E. Zurita, por los datos brindados. A Álvaro Mones, por el aporte bibliográfico. A Rodolfo Salas Gismondi, encargado de la colección de paleontología vertebrados del Museo de Historia Natural de Lima, por el ingreso del material. A Noelia Illarreguy, por la colaboración con el abstract. A los revisores, Laura E. Cruz y Graciela Esteban, y al Comité editor por enriquecer el trabajo.

\section{BIBLIOGRAFÍA}

Ameghino, F. (1889). Contribución al conocimiento de los mamíferos fósiles de la República Argentina. Actas Academia Nacional de Ciencias de Córdoba 6: 32 + 1028 y Atlas de 98 láms.

Burmeister, G. (1866). Lista de los mamíferos fósiles del terreno diluviano. Anales del Museo Público de Buenos Aires 1. pp. 121-232.

Burmeister, H. (1870-1874). Monografía de los glyptodontes en el Museo Público de Buenos Aires. Anales del Museo Público de Buenos Aires 2, pp. 367-377.

Carlini, A. A., Zurita, A. E. y Aguilera, 0. (2008) North American Glyptodontines (Xenarthra, Mammalia) in the Upper
Pleistocene of Northern South America. Palaeontologische Zeitschrift 82 (2), pp. 139-152.

Carranza-Castañeda, O. y Miller, W. E. (2004) Late Tertiary terrestrial mammals from Central Mexico and their relationship to South American immigrants. Revista Brasileira de Paleontologia 7 , pp. 249-261.

Castellanos, A. (1942) A propósito de los géneros Plohophorus, Nopachthus y Panochthus (3ra. parte). Publicaciones del Instituto de Fisiografía y Geología XI, pp. 413-592.

Fariña, R. A. [2001) Física y Matemáticas para reconstruir la vida en el pasado. Actas de Fisiología 6, pp. 45-70.

Gilette, D. D. y Ray, C. E. (1981) Glyptodonts of North America. Smithsonian Contributions to Palaeobiology 40, pp. 1-251.

Gray, J. E. (1869) Catalogue of carnivorous, pachydermatous, and edentate Mammalia in the British Museum. London, British Museum, 398 pp.

Hoffsfetter, R. (1973) Vertebrados cenozoicos y mamíferos cretácicos del Peru. 4to Congreso Latinoamericano de Zoología, Caracas, 10-16 noviembre 1968. Actas 2, pp. 971-983.

Hoffsfetter, R. (1986) High Andean mammalian faunas during the Plio-Pleistocene. En: Vuilleumier, F. y Monasterio, M. (eds.), High altitude tropical biogeography 9, pp. 219-245. New York. Oxford University Press.

Kalafatovich, V. (1955) El fósil gliptodonte hallado en el Cuzco. Revista de la Universidad San Antonio Abad del Cuzco 108, pp. 150-156.

Moreira, L. E. (1965) Notas previas sobre nova espécie de mamífero fóssil do Estado do Caerá, Brasil. Hy Hy Té 2, pp. 41-49.

Oliveira, E. V., Porpino, K. O. y Barreto, A. L. (2010) On the presence of Glyptotherium in the Late Pleistocene of Northeastern Brazil, and the status of Glyptodon and Chlamydotherium. Neues Jahrbuch für Geologie und Palaontologie 258 (3), pp. 353-363.

Owen, R. (1845) Descriptive and illustrated catalogue of the fossil organic remains of Mammalia and Aves. London: Museum of the Royal College of Surgeons of London, $391 \mathrm{pp.}$

Porpino, K. O., Fernícola, J. C. y Bergqvist, L. P. (2010) Revisting the intertropical brazilian species Hoplophorus euphractus (Cingulata, Glyptodontoidea) and the phhylogenetic affinities of Hoplophorus. 
Journal of Vertebrate Paleontology 30 (3), pp. 911-927.

Porpino, K. O., Fernicola, J. C., Cruz, L.E. y Bergqvist, L. P. (2014) The intertropical Brazilian species of Panochthus (Xenarthra, Cingulata, Glyptodontoidea): a reappraisal of their taxonomy and phylogenetic affinities. Journal of Vertebrate Paleontology 34(5), pp 1165-1179.

Pujos, F. y Salas, R. [2004] A Systematic reassessment and paleogeographic review of fossil Xenarthra from Peru. Bulletin de l'Institut Francais d'Etudes Andines 33, pp. 331-377.

Scillato-Yané, G. J. (1977) Sur quelques Glyptodontidae nouveaux (Mammalia, Edentata) du Déséadien (Oligocéne inférieur) de Patagonie (Argentine). Bulletin du Muséum National d'Histoire Naturelle 487, pp. 249-262.

Scillato-Yané, G. J. y Carlini, A. A. (1998) Un Gigantesco Gliptodonte en los Alrededores de la Ciudad de La Plata. Revista Museo de La Plata 11 (2), pp. 45-48.

Scillato-Yané, G. J., Carlini, A. A., Vizcaíno, S. F. y Ortiz-Jaureguizar, E. (1995) Xenarthra. En: Alberdi, M. T., Tonni, E. P. y Leone, G. (eds). Evolución biológica y climática de la región Pampeana durante los últimos cinco millones de años. Un ensayo de correlación con el Mediterráneo Occidental. España, Monografías de la CSIC, pp. 183-209.

Soibelzon, L. H, Zamorano, M., Scillato-Yané, G. J., Piazza, D., Rodríguez, S., Soibelzon, E., Tonni, E. P, San Cristóbal, J. y Beilinson E. (2012) Un Glyptodontidae de gran tamaño en el Holoceno temprano de la región Pampeana de Argentina. Revista Brasileira de Paleontologia 15 [1], pp. 105-112.

Tauber, A. A. y Palacios, M. E. (2007) Nuevos registros de mamíferos cuaternarios de gran porte en la provincia de Santa Cruz, República Argentina. Ameghiniana 44 (4): pp. 41R.

Tejada-Lara, J. V., Salas-Gismondi, R., Pujos, F., Baby, P., Benammi, M., Brusset, S., De Franceschi, D., Espurt, N., Urbina, M. y Antoine, P. O. (2015) Life in Proto-Amazonia: Middle Miocene mammals from the Fitzcarrald Arch (Peruvian Amazonia). Palaeontology 58 (2), pp. 341-378.

Vizcaíno, S. F., Cassini, G. H., Fernícola, J. C. y Bargo, S. (2011) Evaluating habitats and feeding habits through ecomorphological features in glyptodonts (Mammalia, Xenarthra). Ameghiniana 48 (3), pp. 305-319.
Willard, B. (1966) The Harvey Bassler Collection of Peruvian Fossils. Bethlehem, lehigh University, $255 \mathrm{pp}$.

Zamorano, M. (2012) Los Panochthini (Xenarthra, Glyptodontidae): sistemática y evolución. Tesis Doctoral inédita. Universidad Nacional de La Plata.

Zamorano M. (2013) Diagnosis y nueva descripción de Propanochthus bullifer (Burmeister) (Xenarthra, Glyptodontidae). Consideraciones bioestratigráficas y cronológicas de su procedencia. Spanish Journal of Palaeontology 28 (2), pp 285-294.

Zamorano, M. y Brandoni, D. (2013) Phylogenetic analysis of the Panochthini (Xenarthra, Glyptodontidae), with remarks on their temporal distribution. Alcheringa 37, pp. 442-451.

Zamorano, M., Scillato-Yané, G. J., González Ruiz, L. R. y Zurita, A. E. (2011) Revisión de los géneros Nopachtus Ameghino y Phlyctaenopyga Cabrera (Xenarthra, Glyptodontidae, Hoplophorinae) del Mioceno tardío y Plioceno de Argentina. Revista del Museo Argentino de Ciencias Naturales Bernardino Rivadavia 13 (1), PP. 59-68.

Zamorano, M., Scillato-Yané, G. J. y Zurita, A. E. (2013) An enigmatic and large-sized specimen of Panochthus [Glyptodontidae, Panochthini) from the Ensenadan (Early-Middle Pleistocene) of the Pampean region, Argentina. Revista Mexicana de Biodiversidad 84, pp. 847-854.

Zamorano M., Scillato-Yané, G. J. y Zurita, A. E. (2014a) Revisión del genero Panochthus (Xenarthra, Glyptodontidae). Revista del Museo de La Plata 14, pp. 1-46.

Zamorano, M., Taglioretti, M., Zurita, A. E., Scillato-Yané, G. J. y Scaglia, F. (2014b) El registro más antiguo de Panochthus (Xenarthra, Glyptodontidae). Estudios Geológicos 70 (1): eO04 doi: 10.3989/ egeol.41443.289.

Zamorano, M., Oliva, C., Scillato-Yané, G. J., Ferreira, J. D. y Zurita, A. E. (2015) Primer registro de Panochthus greslebini Castellanos (Xenarthra, Glyptodontidae, Hoplophorini) para la región Pampeana. Revista Brasileira de Paleontologia 18 (3), pp. 555-564.

Zurita, A. E. (2007) Sistemática y evolución de los Hoplophorini (Xenarthra, Glyptodontidae, Hoplophorinae. Mioceno tardío-Holoceno tempranoJ. Importancia bioestratigráfica, paleobiogeográfica y paleoambiental. Tesis Doctoral inédita. Universidad Nacional de La Plata. 
Zurita, A. E. y Ferrero, B. (2009) Una nueva especie de Neuryurus Ameghino (Mammalia, Glyptodontidae) en el Pleistoceno tardío de la Mesopotamia de Argentina. El registro más completo del género fuera de la región Pampeana. Geobios 42(5), pp. 663-673.

Zurita, A. E., Miño-Boilini, A. R., Soibelzon, E., Carlini, A. A. y Paredes-Ríos, F. (2009) The diversity of Glyptodontidae (Xenarthra, Cingulata) in the Tarija Valley (Bolivia): systematic, biostratigraphic and paleobiogeographic aspects of a particular assemblage. Neues Jahrbuch für Geologie und Palaontologie 251 (2), pp. 225-237.

Zurita, A. E., Zamorano, M., Scillato-Yané, G. J., González-Ruiz, L. R., Rivas Durán, B. y Céspedez Paz, R. (2011) An exceptional Pleistocene specimen of Panochthus Burmeister (Xenarthra, Glyptodontoidea) from Bolivia: its contribution to the understanding of the early-middle Pleistocene Panochthini. Comptes rendus Palevol 10, pp. 655-664.

Zurita, A. E., Miño-Boilini, A. R., Francia, A. y Arenas-Mosquera, J. E. (2012) The Pleistocene Glyptodontidae Gray, 1869 (Xenarthra: Cingulata) of Colombia and some considerations about the
South American Glyptodontinae. Revista Brasileira de Paleontologia 15 (3), pp. 273-280.

Zurita, A. E., Scillato-Yané, G. J., Ciancio, M., Zamorano, M. y González Ruiz, L. R. (2016) Los Glyptodontidae (Mammalia, Xenarthra): historia biogeográfica y evolutiva de un grupo particular de mamíferos acorazados. En: Agnolin, F. L., Lio, G. L., Brisson Egli, F., Chimento, N. R. y Novas F. E. (Eds). Historia evolutiva y paleobiogeográfica de los vertebrados de América del Sur. XXX Jornadas Argentinas de Paleontología de Vertebrados. Contribuciones del MACN 6, PP 249-262.

Zurita, A. E., Zamorano, M., Scillato-Yané, G. J., Fidel, S., Iriondo, M. y Gillette, D. (2017) A new species of Panochthus Burmeister (Xenarthra, Cingulata, Glyptodontidae) from the eastern cordillera, Bolivia. Historical Biology doi: 10. 1080/08912963.2016.12784.

Zurita, A. E., Gillette, D. D., Cuadrelli, F. y Carlini, A. A. [2018) A tale of two clades: Comparative study of Glyptodon Owen and Glyptotherium Osborn (Xenarthra, Cingulata, Glyptodontidae). Geobios https://doi.org/10.1016/ j.geobios.2018.04.004 